\title{
Obtaining of a PET/SiC hybrid multifilament: Non-isothermal crystallization studies
}

\author{
Marta Riba-Moliner (D), Gabriela Mijas, Davinia Roig and Diana Cayuela \\ INTEXTER, Universitat Politècnica de Catalunya (UPC), Terrassa, Spain
}

ABSTRACT

Thermal processing properties are a key factor in the industrial processing industry. The obtaining of a PET-based hybrid composite multifilament with textile properties (yarns) is presented. The system is composed by ceramic silicon carbide $(\mathrm{SiC})$ nanoparticles and a dispersing agent included in a PET matrix. With the objective to produce a suitable nanocomposite, the study is divided in three parts: (i) evaluation by non-isothermal crystallization of three different dispersing agents (two of them based on esters of montanic acids and, the other on an amide wax) to achieve an homogeneous nanocomposite; (ii) study of the influence of the concentrations of SiC and of the more suitable dispersing agent on the thermal properties of the PET-hybrid composite; and (iii) the obtaining of a PET-hybrid multifilament with textile properties together with its characterization by Gel Permeation Chromatography (GPC), Differential Scanning Calorimetry (DSC) and mechanical properties.
ARTICLE HISTORY

Received 2 July 2020

Accepted 21 October 2020

\section{KEYWORDS}

SiC nanoparticles; PET nanocomposites; nonisothermal kinetics; textile composite; multifilament yarn; dispersing agent

\section{Introduction}

The pursuit of the increase of thermal and mechanical properties of polymeric fibres through composite materials has been an important target in the field of high performance textiles. Optically active particles to produce modulatedemissive fabrics for thermal responsive textiles (Pooley et al., 2016; Shin \& Park, 2018) or biomimetic particles for developing reinforced textile-based tissue engineered scaffolds (Xue et al., 2019), are some of those examples.

In this context, studies of the inclusion of ceramic nanoparticles of titanium dioxide $\left(\mathrm{TiO}_{2}\right)$ and calcium fluoride $\left(\mathrm{CaF}_{2}\right)$ as fillers in a matrix of poly(ethylene terephthalate) (PET) were previously developed by the research group (Cayuela et al., 2016; Manich et al., 2015; Riba-Moliner et al., 2020). In those studies, the use of three additives as dispersing agents was tested. Two esters of montanic acids, one with multifunctional alcohols (MAWMA) and another one partially saponified with butylene glycol and the rest with calcium hydroxide (PSEMA) and, an amide wax based on $\mathrm{N}, \mathrm{N}^{\prime}$-bisstearoylethylene-diamine (AW) were the analysed additives. In the both cases of ceramic-based composite systems, MAWMA agent showed the most suitable behaviour in terms of non-isothermal crystallization, dispersion degree and compatibility, which are important parameters from the industrial processing point of view (Riba-Moliner et al., 2020).

In the present work, $\mathrm{SiC}$ is studied as filling material for the production of a multifilament with textile properties. $\mathrm{SiC}$ is one of the most important carbide ceramics and, although it is manufactured in different varieties, all of them show similar properties. From high to very high mechanical resistance, corrosion resistance even at very high temperatures, high thermal resistance, low thermal expansion, very high thermal conductivity, significant hardness and semiconductivity are some of the most relevant and interesting properties of this ceramic material (Agarwal et al., 2013; Bhattacharjee \& Nanda, 2017; Deng et al., 2018; Mullaikodi et al., 2019; Patnaik \& Nayak, 2018; Shen et al., 2017).

The objective of this study is to produce a hybrid composite filament based on PET filled with SiC particles and a dispersing agent to ensure both, homogeneous mixtures and final properties, avoiding the aggregation of the ceramic part. Firstly, non-isothermal crystallization studies of the nanocomposited system ( $\mathrm{PET} / \mathrm{SiC})$ with different dispersing agents (MAWMA, PSEMA and AW) are developed by DSC in order to determine the contribution of each one in the crystallization process. Once the more promising nanocomposited (NCP) system is found, a study of the influence of the concentrations of $\mathrm{SiC}$ and dispersing agent to the thermal properties of the NCP in plate form is determined by DSC. Finally, the $\mathrm{PET} / \mathrm{SiC} /$ dispersing agent system obtained at the more favourable conditions is transformed into a multifilament with textile properties by extrusion. Molecular weight determination, thermal and mechanical properties are evaluated by Gel Permeation Chromatography (GPC), DSC and mechanical tests, respectively, to characterize those resulting substrates.

\section{Experimental part}

\subsection{Materials}

\subsubsection{Poly(ethylene terephthalate) (PET)}

PET textile quality was used in the determination of the suitability of the dispersing agent to produce composites 
Table 1. Nano-composite samples with different SiC and dispersing agent concentrations.

\begin{tabular}{lcc}
\hline $\begin{array}{l}\text { Name of } \\
\text { the sample }\end{array}$ & $\begin{array}{c}\mathrm{SiC} \\
\text { concentration (\%) }\end{array}$ & $\begin{array}{c}\text { Dispersing agent } \\
\text { concentration (\%) }\end{array}$ \\
\hline N1.2 & 1 & 2 \\
N1.4 & 1 & 4 \\
N2.2 & 2 & 2 \\
N2.4 & 2 & 4 \\
N3.2 & 3 & 2 \\
N3.4 & 3 & 4 \\
\hline
\end{tabular}

and was provided by ANTEX, S.L. 100\% polyester (extrabright). PET bottle grade, was used to determine the effect of the concentration of silicon carbide and dispersing agent and was provided by La Seda de Barcelona, Artenius Flow 100\% PET without additives (bright).

Moreover, PET pellets were stored in a vacuum oven at $50{ }^{\circ} \mathrm{C}$ and 80 mbar to prevent the degradation and the absorption of humidity of polymer.

\subsubsection{Silicon carbide $(\mathrm{SiC})$ nanoparticles}

$\beta$-SiC spherical nanoparticles, purity of $97.5 \%$, average particle size of $45-55 \mathrm{~nm}$ and specific surface area (BET) 35$40 \mathrm{~m}^{2} / \mathrm{g}$ were purchased to NanoAmor.

\subsubsection{Dispersing agents}

All dispersing agents were supplied by Clariant. Ester of montanic acids with multifunctional alcohols (MAWMA) LICOWAX E flakes, ester of montanic acids partly saponified with butylene glycol and the rest with calcium hydroxide (PSEMA) LICOWAX OP powder and amide wax based on N,N'-Bis-stearoylethylene-diamine (AW) LICOWAX $\mathrm{C}$ powder.

\subsection{Composites preparation}

\subsubsection{PET/SiC composite}

Nano-silicon carbide was used to prepare composites aiming the evaluation of dispersing agents. Substrates were prepared without and with $(2 \%)$ of $\mathrm{SiC}$ particles in the presence and in the absence of the different dispersing agents (5\%). Mixtures were extruded using a Corima extruder Model G132 A. The conditions for the extrusion were $275^{\circ} \mathrm{C}$ and a screw speed of $70 \mathrm{rpm}$. Resulting composites were stored at $105^{\circ} \mathrm{C}$ for $24 \mathrm{~h}$.

In a further step, the influence of the ratio SiC:dispersing agent concentration in the composite properties was evaluated. The dispersing agent used was the one that showed the most favourable non-isothermal crystallization behaviour (Table 1, Sánchez-Leija et al., 2014).

\subsubsection{Multifilament yarn}

A PET multifilament yarn containing $2 \%$ nano-metric $\mathrm{SiC}$ and $2 \%$ dispersing agent, was prepared and supplied by IQAP Masterbatch Group S.L. The yarn was subsequently stretched at $190^{\circ} \mathrm{C}$ at a drawn ratio $1: 2$ in a laboratory stretching equipment.

\subsection{Characterization}

\subsubsection{Differential scanning calorimetry (DSC)}

Thermal analysis tests were performed in a Differential Scanning Calorimeter DSC 7 Perkin Elmer. $7.5 \mathrm{mg}$ of sample was sealed in an aluminium crucible. Temperature program was set according to the experiment developed. Samples were tested by duplicated and results show the mean of them. Non-isothermal crystallization studies to determine the more suitable dispersing agent were developed in the following conditions: (i) heat up from $40{ }^{\circ} \mathrm{C}$ to $290{ }^{\circ} \mathrm{C}$ at $300^{\circ} \mathrm{C} / \mathrm{min}$, (ii) holding at $290^{\circ} \mathrm{C}$ for $5 \mathrm{~min}$, (iii) cool down from $290^{\circ} \mathrm{C}$ to $40^{\circ} \mathrm{C}$ at $5,10,15$, and $20^{\circ} \mathrm{C} / \mathrm{min}$. Thermal properties of yarns were determined according heating up from $40{ }^{\circ} \mathrm{C}$ to $300{ }^{\circ} \mathrm{C}$ at $20^{\circ} \mathrm{C} / \mathrm{min}$. Nitrogen $\left(\mathrm{N}_{2}\right)$ purge gas at a flow of $35 \mathrm{ml} / \mathrm{min}$ was used for all DSC experiments.

\subsubsection{Molecular weight}

Yarns were previously washed with Sandozina NIA (Clariant) $1 \mathrm{~g} / \mathrm{L}$ at $35 \pm 2{ }^{\circ} \mathrm{C}$ for $30 \mathrm{~min}$ and with deionized water at room temperature for $10 \mathrm{~min}$ three times. Molecular weight determination followed the procedure described in Gacén Esbec (2004). Approximately, $3 \mathrm{mg}$ of washed yarns were placed in a test tube with $0.2 \mathrm{~mL}$ of $\mathrm{o}-$ chlorophenol (>99\%, Aldrich). The tubes were placed in a polyethylene glycol bath at $80^{\circ} \mathrm{C}$ for $30 \mathrm{~min}$. Once the yarn was dissolved and the tubes cooled, $1.8 \mathrm{~mL}$ of chloroform (HPLC grade, stabilized with amylene, approx. $150 \mathrm{ppm}$, Scharlau) was incorporated. The mixture was stirred and subsequently, the solution was filtered (PTFE filter, $0.45 \mu \mathrm{m}$ ) and transferred to a vial. Analysis was carry out in high performance liquid chromatograph (Perkin Elmer) provided with Injector model ISS200, 200lc series pump, oven for columns series 200, refractive index detector Series 200a. The separation was performed at $40^{\circ} \mathrm{C}$ with chloroform (HPLC grade, Scharlau) at a flow rate of $1 \mathrm{~mL} / \mathrm{min}$ on PLgel $5 \mu \mathrm{m}$ Mixed-D column $300 \times 7.5 \mathrm{~mm}$ (Agilent) proceeded by Oligopore Column $6 \mu \mathrm{m} 300 \times 7.5 \mathrm{~mm}$ (Agilent). The system was calibrated with polystyrene standards with weight average molecular weight in the range $2350-200,000 \mathrm{~g} / \mathrm{mol}$ (Waters). Software TotalChrom Navigator - TurboSEC v. 6.3.1.0504 was used to calculate molecular weight in number, molecular weight in weight and polydispersity.

\subsubsection{Lineal density}

Before the test, yarns were conditioned according to UNEEN 13392: 2001. For the determination of the count, $100 \mathrm{~m}$ of each yarn was collected in the Aspe JBA. Subsequently, samples were weighted on an analytical balance $( \pm 0.1 \mathrm{mg}$ ) and the relationship between mass and length was calculated. This procedure was performed for each sample by triplicate.

\subsubsection{Tenacity and elongation}

Tensile tests were carried out following the procedure described in UNE-EN ISO 2062: 2010. Uster Tensokid 
automatic dynamometer, model PE 4056 was used. The testXpert Standard v 6.01 software was used to obtain the stress-strain curves. System was set up to an elongation of $85 \%$ of the initial test piece.

\section{Theoretical background}

\subsection{Isothermal crystallization}

The analysis of the crystallization kinetics by Avrami's model (Avrami, 1939, 1940, 1941) relates the degree of relative crystallinity with time, when the process occurs at a constant temperature of crystallization (Equation 1).

Equation

$$
1-\chi(t)=\exp \left[-Z_{t} \cdot\left(t-t_{0}\right)^{n}\right]
$$

where $\chi(t)$ is the relative degree of crystallinity, $n$ is the Avrami's coefficient or exponent which value is dependent on the crystallization mechanism (determines the nucleation and growth modes), and $Z_{t}$ is a constant parameter which units depend on $n$. Thus, $Z_{t}$ parameter is not suitable for comparing kinetics of processes with uneven nucleation evolutions. In those scenarios, the use of a normalized rate constant parameter $(k)$, defined as $k=Z_{t}{ }^{1} / n$, allows to work in time $^{-1}$ units, which are independent of the $n$ value. Then, the $n$ value can be calculated and the type of nucleation (instantaneous/spontaneous, homogeneous/heterogeneous) and geometry $(1 \mathrm{D}, 2 \mathrm{D}$ or $3 \mathrm{D})$ of the growth can be approached according to a normalized table.

It is worth mentioning that the definition of $Z_{t}$ and $n$ parameters differ in isothermal and non-isothermal crystallization. In both cases, the cooling rate affects the rate of nuclei formation and growth of spherulites.

\subsection{Non-isothermal crystallization}

The evolution of non-isothermal crystallization processes can be followed performing experiments either or from the vitreous state of the polymer (below the glass transition temperature) using different heating rates, or from the melted sample using with different cooling rates. However, the last is more studied because approaches more to a simulated processing conditions. According to this, non-isothermal crystallization processes are based on the evolution of the crystallinity degree with the temperature at constant heating or cooling rates. In this sense, Jeziorny modified Avrami's model to describe the non-isothermal crystallization (Equation 2).

$$
\chi=1-\exp \left(Z_{t} \cdot t^{n}\right)
$$

This correction allowed the extrapolation of Avrami's model parameters, where the $n$ exponent is a constant mechanism (with variations from 1 to 4 ). $n$ value is depending on the type of nucleation (homogeneous or heterogeneous) and on the growing process parameters $(1 \mathrm{D}, 2 \mathrm{D}$ or $3 \mathrm{D})$. On the other hand, $Z_{t}$ corresponds to a rate constant that includes both, the nucleation parameter and the growth rate. By developing Equations (2) and (3) appears.

$$
\ln [-\ln (1-\chi)]=n \cdot \ln t+\ln \cdot Z_{t}
$$

In order to determine the kinetic parameters of $n$ and $Z_{t}, \ln -\ln v s . \ln t$ is plotted. Jeziorny characterized non-isothermal crystallization by correcting the Avrami's model parameters (Equation 4).

$$
\ln \mathrm{Z}_{\mathrm{c}}=\frac{\ln \mathrm{Z}_{\mathrm{t}}}{\alpha}
$$

where $\alpha$ corresponds to the cooling rate. The conversion degree and the corresponding kinetic parameters are obtained from the DSC thermograms by the calculation of the partial areas of the exothermic crystallization peak (Equation 5).

$$
\chi(\mathrm{t})=\frac{\int_{0}^{\mathrm{t}}\left(\frac{\mathrm{dH}}{\mathrm{dt}}\right) \mathrm{dt}}{\int_{0}^{\infty}\left(\frac{\mathrm{dH}}{\mathrm{dt}}\right) \mathrm{dt}}=\frac{\Delta \mathrm{H}_{\mathrm{t}}}{\Delta \mathrm{H}_{\infty}}
$$

where $\chi(t)$ is the degree of crystallization at each cooling rate, $t$ parameter is time and $\frac{\Delta H_{t}}{\Delta H_{\infty}}$ corresponds to the partial exotherm of crystallization. The degree of crystallization at each cooling rate is determined by the integration of the partial crystallization exotherm, previously delimited. Delimitation is set by referencing the time at which crystallization begins $\left(\mathrm{t}_{0}\right)$.

\section{Results and discussion}

\subsection{Effect of dispersing agents on SiC NCPs}

With the aim to obtain $\mathrm{PET} / \mathrm{SiC}$ fibres with textile properties (yarns), firstly blends of $\mathrm{PET} / \mathrm{SiC} /$ dispersing agent were studied towards the elucidation of the more appropriate conditions for an optimum composite. In order to achieve that, the evaluation of the dispersing effect of the ceramic nanoparticles (NPs) into the matrix with different dispersing agents (MAWMA, PSEMA and AW) was carried out. Nonisothermal crystallization behaviour of the nanocomposites (NCPs) with and without the presence of the dispersing agents at different cooling rates was carried out by Differential Scanning Calorimetry (DSC) technique. To distinguish between the effect of the dispersing agents and NPs and their mixtures, blends of PET/dispersing agent were prepared, analysed and compared with the NCP homologous. In all cases, the evolution of crystallization temperatures ( $\mathrm{Tc}$ ) showed a decrease when the cooling rates increased (Figure 1). The incorporation of dispersing agent to PET was translated in an increase of Tc values respect pure polymer and, at the same time, below of PET/SiC values, revealing different nucleating behaviour depending on the dispersing agent used. In this way, MAWMA showed the most intense nucleating nature, followed by PSEMA and finally AW, which was the less and, even exhibited Tc values below pure polymer at cooling rates above $10^{\circ} \mathrm{C} / \mathrm{min}$.

Significant changes were spotted when SiC NPs were incorporated to the systems (Figure 1). Contrastingly to the previous samples, nucleating behaviour of the NCP systems changed when $\mathrm{SiC}$ was added, being NCP/AW the sample that more favoured the nucleation of polyester, followed by 


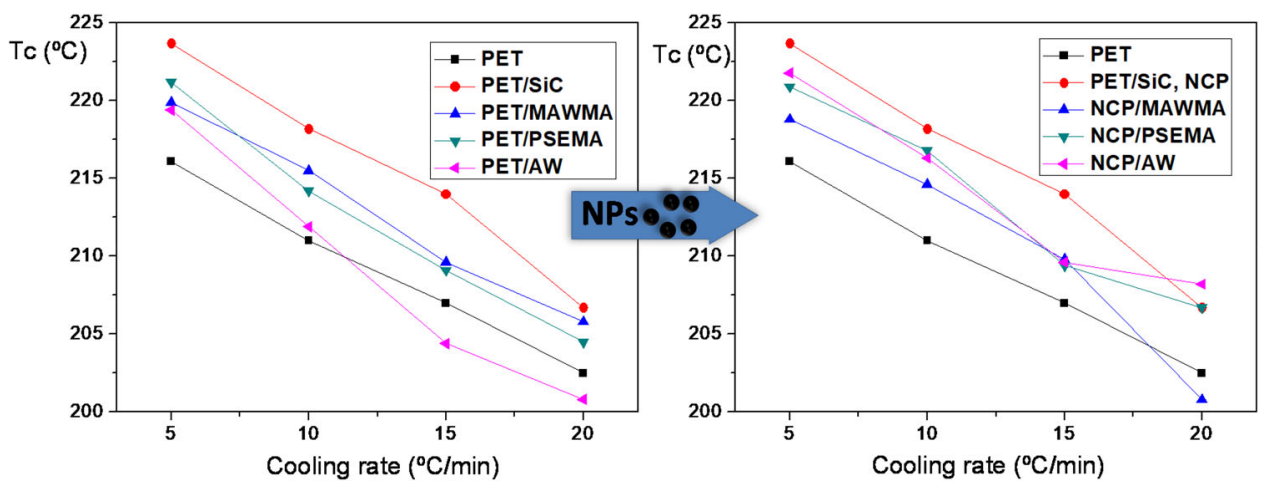

Figure 1 Evolution of Tc with cooling rates of (left) PET, PET/SiC and PET/dispersing agent samples and (right) PET, PET/SiC and PET/SiC/dispersing agent NCPs.

Table 2. Mean values and standard deviation of $\Delta \mathrm{Hc}$, for all the cooling rates, for all the samples.

\begin{tabular}{lcccc}
\hline & \multicolumn{4}{c}{$\Delta H \mathrm{Hc}(\mathrm{J} / \mathrm{g})$} \\
\cline { 2 - 5 } SiC content & PET & PET + PSEMA & PET + MAWMA & PET + AW \\
\hline $\mathbf{0} \%$ & $-56.3 \pm 1.7$ & $-56.6 \pm 0.4$ & $-55.4 \pm 2.2$ & $-54.3 \pm 2.6$ \\
$\mathbf{2} \%$ & $-48.7 \pm 1.5$ & $-49.8 \pm 2.8$ & $-52.5 \pm 2.2$ & $-52.3 \pm 1.2$ \\
\hline
\end{tabular}

NCP/PSEMA and finally NCP/MAWMA. Furthermore, NCP/PSEMA and NCP/AW increased the Tc values respect their homologues without NPs, while NCP/MAWMA did not show substantial changes in this way. This observation may indicate that SiC NPs would drive the non-isothermal crystallization of PSEMA and AW composites; however, in the case of mixtures with MAWMA, the dispersing agent would guide the process by itself. Thus, an increase of the degree of dispersion and compatibility between the organic fraction (PET) and the inorganic $(\mathrm{SiC})$ and, consequently, the obtaining of a more homogeneous composite due to the incorporation of MAWMA could be concluded. Similar conclusions were found in a previous work developed by the group for fluorite $\left(\mathrm{CaF}_{2}\right) \mathrm{NPs}$ (Riba-Moliner et al., 2020). In that study, NCP/PSEMA and NCP/AW exhibited crystallization properties similar to PET/PSEMA and PET/ AW, respectively, however NCP/MAWMA revealed characteristics nearly to neat PET. The lipophilic character of MAWMA provided major chemical compatibility between PET and NPs, resulting in an environment of the composite close to pure polymer. Thus, NCP/MAWMA exhibited a non-isothermal crystallization behaviour more similar to pure PET rather the rest (NCP/PSEMA and NCP/AW), whose were more nearby to $\mathrm{PET} / \mathrm{SiC}$ system. For this reason and after observing NCP/MAWMA non-isothermal evolution, a higher effect of compatibility of MAWMA on $\mathrm{SiC}$ NPs into PET matrix over PSEMA and AW was presumably attributed.

Furthermore, the evolution of cold crystallization enthalpies $(\Delta \mathrm{Hc})$ for PET/dispersing agent and NCP samples were spotted in concordance with Tc results (Table 2). Due to $\Delta \mathrm{Hc}$ values at the different cooling rates resulted very similar, mean values were analysed. A relevant decrease of $\Delta \mathrm{Hc}$ in absolute value when NPs were added to PET at all cooling rates for all samples was revealed. The inclusion of $\mathrm{SiC}$ decreased the polymer fraction able to crystallize, affecting the $\Delta \mathrm{Hc}$ parameter. However, no significant differences were observed between $0 \% \mathrm{SiC}$ samples and between $2 \% \mathrm{SiC}$ homologues, so, no clear correlation could be established.

Consecutively, the evolution of the crystallization $(\chi)$ for the NCP systems was determined for each system by calculating the partial areas of the exothermic crystallization peak over the integration of the crystallization peak (Equation 5). $\chi$ values of the different cooling rates revealed similar tendencies in all NCP systems (Figure 2). Z- and $S$-shaped tendencies were determined for the percentage of transformation with temperature and time, respectively.

Afterwards, Avrami's kinetic parameter $n$ was calculated for all $\mathrm{NCP} /$ dispersing agent samples at rates of 5, 10, 15 and $20^{\circ} \mathrm{C} / \mathrm{min}$ through Equation (3) (Figure 3). Indeed, polymer and NCP samples depicted almost linear evolutions, corroborating that NCPs followed a crystallization process according to Avrami's model (Avrami, 1940). In the case of $\mathrm{PET} / \mathrm{SiC}$ sample, values whose slightly deviated from linearity where avoided in the calculation of $n$. The corresponding values of the slopes of the different systems ( $n$ values) and resulting $Z t$ values are listed in Tables 3 and Cayuela et al. (2016). The incorporation of NPs to PET (PET/SiC) did not modify significantly $n$ values which quietly increased, excepting for rates of $15^{\circ} \mathrm{C} / \mathrm{min}$, suggesting that the crystallization processes occurred generally through heterogeneous spherulites (Avrami, 1940) growing in $3 \mathrm{D}(n=1+\mathrm{D})$. The degrees of freedom of the systems and also, the total entropy, increased when dispersing agents were included in the majority of the cases, pointing to a presumably more complex crystallization scenario for NCPs. On the other hand, $Z t$ experienced more evident modifications according to cooling rates and the different samples. $Z t$ increased in all cases in the presence of $\mathrm{SiC}$ compared to pure PET because this parameter is related with the crystallization rate. Thus, faster conversion processes occurred in nanoparticulated systems. From all NCP samples, NCP/ MAWMA exhibited lowest $Z t$ values even at rates of $20^{\circ} \mathrm{C} /$ min. With these observations, it could be concluded that a more homogeneous and improved crystallization process of $\mathrm{PET} / \mathrm{SiC}$ system could be induced when MAWMA was incorporated as dispersing agent (Table 4).

Extensively, the corrected crystallization value, $Z_{c}$, was calculated for all systems through Equation (4). $Z_{c}$ parameter appeared to be almost independent of cooling rates above $5{ }^{\circ} \mathrm{C} / \mathrm{min}$ which was in concordance with its definition (Table 5). 

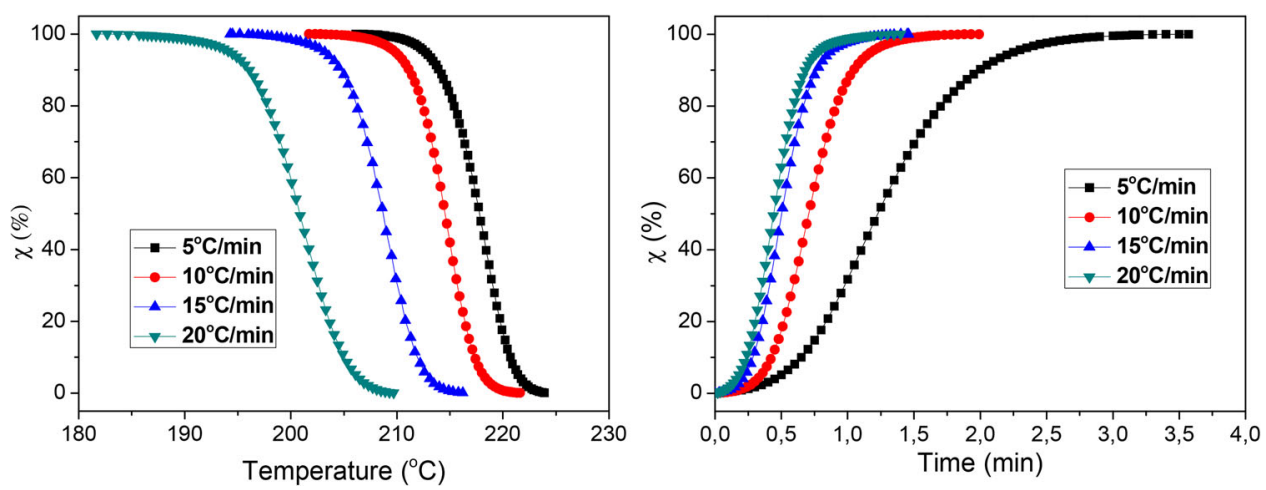

Figure 2. Evolution of $\chi$ of NCP/MAWMA and different cooling rates in front of (left) temperature and (right) time.
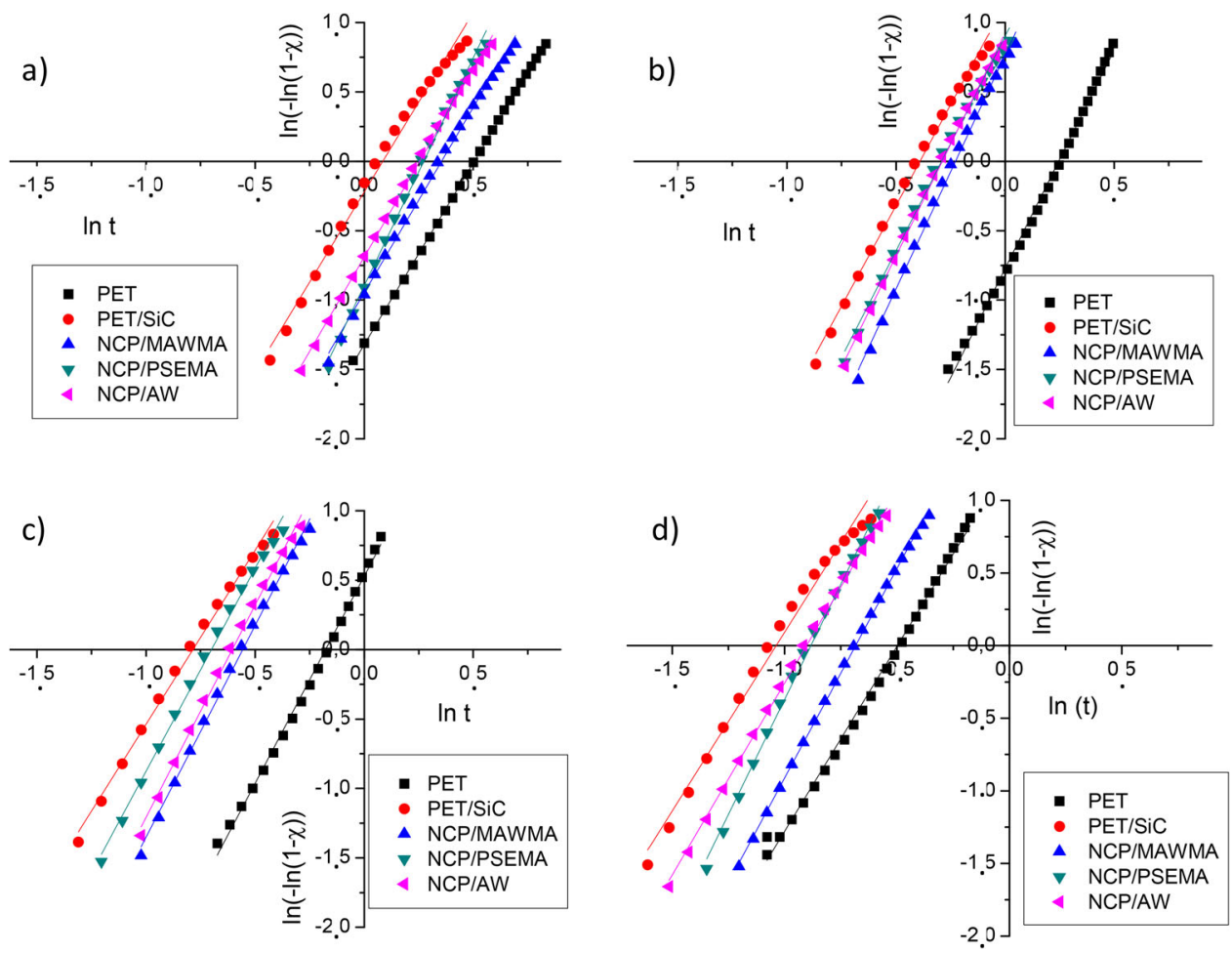

Figure 3. Avrami's relation for NCP/dispersing agent samples at a cooling rates of a) $5^{\circ} \mathrm{C} / \mathrm{min}$, b) $\left.10^{\circ} \mathrm{C} / \mathrm{min}, \mathrm{c}\right) 15^{\circ} \mathrm{C} / \mathrm{min}$ and d) $20^{\circ} \mathrm{C} / \mathrm{min}$. Data was fitted to straight lines to obtain lineal regressions.

Table 3. Avrami's kinetic parameter $n$ of PET, PET/SiC and NCP/dispersing agents at different cooling rates.

\begin{tabular}{lccccc}
\hline$\alpha\left({ }^{\circ} \mathrm{C} / \mathrm{min}\right)$ & $\mathrm{PET}$ & $\mathrm{PET} / \mathrm{SiC}$ & $\mathrm{NCP} / P S E M A$ & $\mathrm{NCP} / M A W M A$ & $\mathrm{NCP} / \mathrm{AW}$ \\
\hline $\mathbf{5}$ & 2.6 & 2.9 & 3.3 & 3.0 & 2.8 \\
10 & 2.9 & 3.0 & 3.3 & 3.3 & 3.2 \\
$\mathbf{1 5}$ & 3.0 & 2.6 & 3.0 & 3.2 & 2.9 \\
$\mathbf{2 0}$ & 2.6 & 2.7 & 3.0 & 3.4 & 2.8 \\
\hline
\end{tabular}

Table 4. Avrami's kinetic parameters $Z_{t}\left(\mathrm{~min}^{-n}\right)$ of PET, PET/SiC and NCP/dispersing agents at different cooling rates.

\begin{tabular}{lccccc}
\hline$\alpha\left({ }^{\circ} \mathrm{C} / \mathrm{min}\right)$ & $\mathrm{PET}$ & $\mathrm{PET} / \mathrm{SiC}$ & $\mathrm{NCP} /$ PSEMA & NCP/MAWMA & NCP/AW \\
\hline 5 & 0.27 & 0.83 & 0.47 & 0.38 & 0.47 \\
10 & 0.65 & 3.40 & 2.89 & 2.25 & 2.86 \\
15 & 1.71 & 8.16 & 6.62 & 5.30 & 5.90 \\
20 & 3.83 & 18.62 & 14.44 & 5.68 & 12.30 \\
\hline
\end{tabular}

Furthermore, half crystallization times $\left(t_{1 / 2}\right)$ were calculated by taking the time where $\chi$ reached $50 \%$ of the total conversion (Figure 4). $\mathrm{t}_{1 / 2}$ decreased when $\mathrm{SiC}$ was
Table 5. Avrami's kinetic parameters $Z_{c}$ of PET, PET/SiC and NCP/dispersing agents at different cooling rates.

\begin{tabular}{lccccc}
\hline$\alpha\left({ }^{\circ} \mathrm{C} / \mathrm{min}\right)$ & $\mathrm{PET}$ & $\mathrm{PET} / \mathrm{SiC}$ & NCP/PSEMA & NCP/MAWMA & NCP/AW \\
\hline 5 & 0.77 & 0.96 & 0.86 & 0.82 & 0.86 \\
10 & 0.96 & 1.13 & 1.11 & 1.08 & 1.11 \\
15 & 1.04 & 1.15 & 1.13 & 1.12 & 1.13 \\
20 & 1.07 & 1.16 & 1.14 & 1.09 & 1.13 \\
\hline
\end{tabular}

incorporated, corroborating the nucleating effect the NPs in the crystallization process. Conversely, NCP/MAWMA system showed different behaviour respect the rest of the NCPs and adopted a value of $0.53 \mathrm{~min}^{1 / 2}$, almost the same as PET when was cooled at $20^{\circ} \mathrm{C} / \mathrm{min}$. This would suggest that NCP/MAWMA follows similar thermal evolution as pure polymer when is cooled fast, which is very relevant in thermal industrial processing of composite materials. 


\subsection{Effect of SiC particle and MAWMA concentrations on NCP}

According to the results of non-isothermal crystallization, NCP/MAWMA system evidenced more promising results from the industrial processing point of view. For this reason, a study of the influence of the $\mathrm{SiC}$ and dispersing agent concentrations on the NCP/MAWMA composite was developed. It is needed to highlight that, those samples were moulded for other proposals and, consequently, the textile PET substrate used in the previous section was replaced by another PET more suitable for moulding.

The determination of the thermal behaviour of the different NCPs was carried out by DSC technique. Prior to this, samples were heated up to $290^{\circ} \mathrm{C}$ to 'clean' its thermal history, then cooled at $20^{\circ} \mathrm{C} / \mathrm{min}$ to record differences in the non-isothermal crystallization behaviour and heated again (Figure 5). With this, variations on the resulting

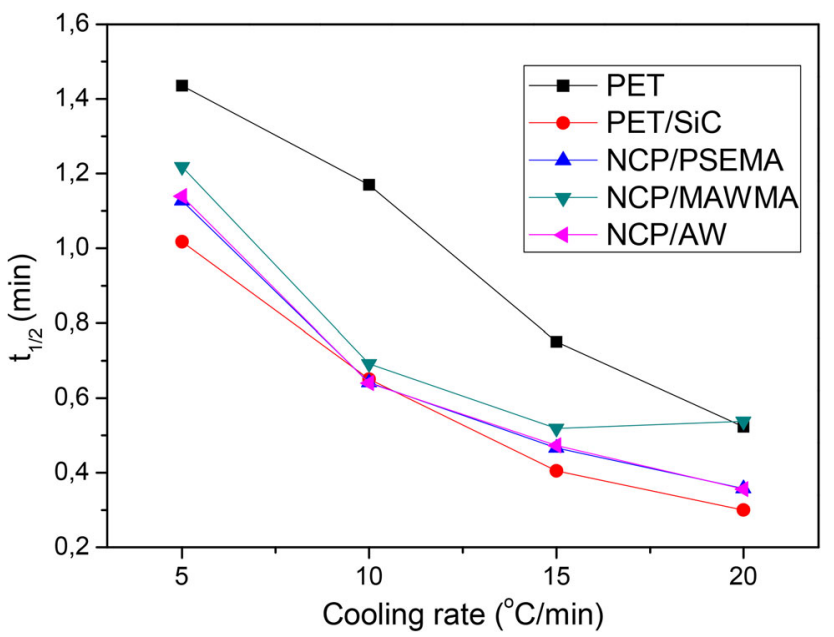

Figure 4. Half crystallization time, $\mathrm{t}_{1 / 2}$, of PET, PET/SiC and NCP/dispersing agent samples at $5,10,15$ and $20^{\circ} \mathrm{C} / \mathrm{min}$. thermograms will be only related with differences in the concentration of $\mathrm{SiC}$ and/or MAWMA.

From the crystallization region of the resulting thermograms, Tc and $\Delta \mathrm{Hc}$ parameters were calculated for all samples (Table 6). The inclusion of NPs increased Tc and absolute $\Delta \mathrm{Hc}$ in all cases. $\Delta \mathrm{Hc}$ did not vary significantly in N1.2, N1.4 and N2.2, but experienced a subtle increase for higher SiC and MAWMA contents. This variation was attributed to a packing effect exerted by the components of the mixture that effected the entropy of the systems, hampering the crystallization process (Serenko et al., 2017). On the other hand, no clear tendency could be extracted with $\Delta \mathrm{Hc}$ values.

Concomitantly, a decrease of the glass transition temperature $(\mathrm{Tg})$ after the inclusion of $\mathrm{SiC}$ was appreciated when glass transition region of thermograms was analysed (Table 7). This decrease was almost insignificant for sample N1.2, which was composed by $1 \%$ of $\mathrm{SiC}$ and $2 \%$ of MAWMA, respect pure PET. In that case, the combination of low NP concentration plus a double-concentrated MAWMA resulted in a well dispersed system with glass transition behaviour close to PET. However, when those concentration values were higher, the decrease of the $\mathrm{Tg}$ was more evident, probably due to the poor-packed macromolecular chains of the polymeric fraction as result of the presence of $\mathrm{SiC}$ and MAWMA molecules into the structure, those facilitating the chain movement (Serenko et al., 2017). The presence of dispersing agent accentuated that behaviour, providing plasticity to the polymer and decreasing the entropy of the system compared to N1.2 sample (Serenko et al., 2017). Thus, higher miscible systems with an induction of a deceleration of the bulk crystallization and an acceleration of the spherulitic growth attributed to higher heterogeneous nucleation rates could be concluded (Krikorian \& Pochan, 2004). Contrarily, heat capacities $(\Delta \mathrm{Cp})$ and melting points $(\mathrm{Tm})$ did not reveal important changes with the content of the NCP. Moreover, the enthalpy corresponding to the melting peak $(\Delta \mathrm{Hm})$ was

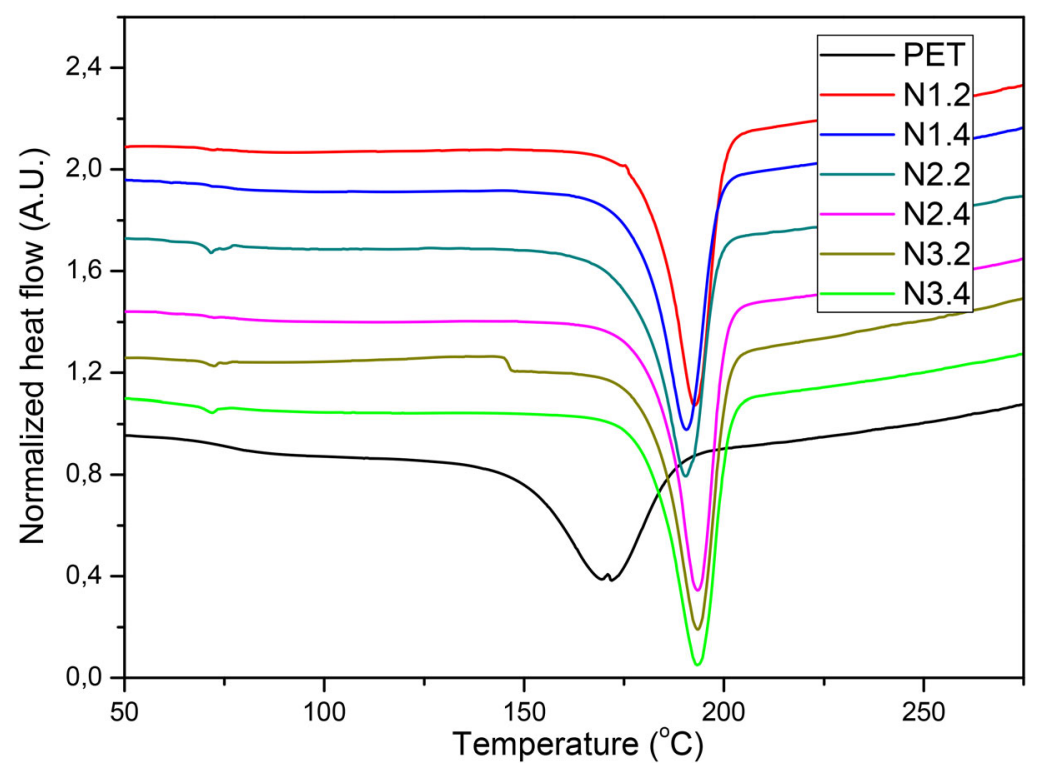

Figure 5. Cold crystallization peaks from DSC thermograms of PET and NCP samples. 
Table 6. Tc and $\Delta \mathrm{Hc}$ values calculated from the non-isothermal crystallization thermograms for all the samples.

\begin{tabular}{lcc}
\hline Sample & Tc $\left({ }^{\circ} \mathrm{C}\right)$ & $\Delta \mathrm{Hc}(\mathrm{J} / \mathrm{g})$ \\
\hline PET & 171.6 & -47.5 \\
N1.2 & 192.6 & -49.9 \\
N1.4 & 190.6 & -49.8 \\
N2.2 & 190.3 & -50.5 \\
N2.4 & 193.6 & -53.4 \\
N3.2 & 193.6 & -48.6 \\
N3.4 & 193.3 & -54.2 \\
\hline
\end{tabular}

Table 7. Thermal properties of PET and NCP/dispersing agent samples obtained from DSC technique.

\begin{tabular}{|c|c|c|c|c|}
\hline \multirow[b]{2}{*}{ Sample } & \multicolumn{2}{|c|}{ Glass transition region } & \multicolumn{2}{|c|}{ Melting region } \\
\hline & $\operatorname{Tg}\left({ }^{\circ} \mathrm{C}\right)$ & $\Delta \mathrm{Cp}\left(\mathrm{J} / \mathrm{g} \cdot{ }^{\circ} \mathrm{C}\right)$ & $\operatorname{Tm}\left({ }^{\circ} \mathrm{C}\right)$ & $\Delta \mathrm{Hm}(\mathrm{J} / \mathrm{g})$ \\
\hline PET & 78.3 & 0.16 & 244.3 & 36.2 \\
\hline $\mathrm{N} 1.2$ & 77.1 & 0.16 & 244.7 & 37.1 \\
\hline N1.4 & 76.7 & 0.16 & 244.7 & 38.2 \\
\hline $\mathrm{N} 2.2$ & 75.1 & 0.16 & 244.3 & 37.6 \\
\hline $\mathrm{N} 2.4$ & 75.3 & 0.16 & 245.7 & 39.4 \\
\hline N3.2 & 74.9 & 0.16 & 245.3 & 39.6 \\
\hline N3.4 & 73.3 & 0.14 & 244.7 & 40.1 \\
\hline
\end{tabular}

corrected taking into account the percentage of polyester in the mixture, nonetheless a clear tendency between the different content of samples could not be established.

In summary, after evaluating the influence of the concentration of SiC and MAWMA to the NCP from the point of view of the crystallization properties, N2.2 sample evidenced the more balanced behaviour. Consequently, NCP mixtures containing $2 \% \mathrm{SiC}$ and 2\% MAWMA into a PET matrix were prepared towards the production of the hybrid multifilament.

\subsection{Sic NCP multifilament}

Once the hybrid NCP/MAWMA multifilament was produced by extrusion, results obtained from molecular weight determination indicated that the number molecular weight $\left(\overline{\boldsymbol{M}_{n}}\right)$ and the weight molecular weight $\left(\overline{\mathrm{M}_{\mathrm{W}}}\right)$ decreased compared to pure PET filament, whereas polydispersity $\left(\overline{\mathrm{M}_{\mathrm{w}}} / \overline{\mathrm{M}_{\mathrm{n}}}\right)$ remained constant (Table 8$)$. This was observed in a previous study carried out by the same research group in which a novel hybrid filament of PET-CaF $\mathrm{C}_{2}$ was developed (Riba-Moliner et al., 2020). It has been found that PET nanocomposites showed a reduction on viscosity, which means that PET nanocomposites are more sensitive to degradation due to the long residence times, friction and high shear heating during the melt-compounding process (Sánchez-Solís et al., 2004; Yang et al., 2010).

On the other hand, with the aim to evaluate thermal properties of NCP/MAWMA, thermal analyses were carried out by DSC. Two melting points $\left(\mathrm{T}_{\mathrm{m} 1}\right.$ and $\left.\mathrm{T}_{\mathrm{m} 2}\right)$ were found in PET and NCP/MAWMA samples at similar temperatures (Table 9). Due to the similarity between them and due to melting temperature is associated to the crystalline fraction of samples (Cheng \& Jin, 2002), the origin of the contribution of the crystalline fraction of NCP was attributed mostly to PET. Moreover, the appearing of two melting points was correlated to the sum of the influence of the spinning and
Table 8. Molecular weight results of PET and NCP/MAWMA multifilament obtained by GPC.

\begin{tabular}{lccc}
\hline Sample & $\bar{M}_{n}(\mathrm{~kg} / \mathrm{mol})$ & $\bar{M}_{w}(\mathrm{~kg} / \mathrm{mol})$ & $\bar{M}_{w} / \bar{M}_{n}$ \\
\hline PET & 27.6 & 51.5 & 2.0 \\
NCP/MAWMA & 20.6 & 37.4 & 2.0 \\
\hline
\end{tabular}

Table 9. Thermal parameters of PET and NCP/MAWMA multifilament obtained by DSC. $\left.{ }^{*} \chi_{c}=\Delta \mathrm{H}_{\text {sample }} / \Delta \mathrm{H}_{\mathrm{PET}}\right) \cdot 100$.

\begin{tabular}{lccccccc}
\hline & \multicolumn{3}{c}{ Heating } & & \multicolumn{3}{c}{ Cooling } \\
\cline { 2 - 4 } \cline { 8 - 9 } Sample & $\mathrm{T}_{\mathrm{m} 1}\left({ }^{\circ} \mathrm{C}\right)$ & $\mathrm{T}_{\mathrm{m} 2}\left({ }^{\circ} \mathrm{C}\right)$ & $\Delta \mathrm{H}_{\mathrm{m}}(\mathrm{J} / \mathrm{g})$ & $\mathrm{T}_{\mathrm{c}}\left({ }^{\circ} \mathrm{C}\right)$ & $\Delta \mathrm{H}_{\mathrm{c}}(\mathrm{J} / \mathrm{g})$ & $\chi_{\mathrm{c}}{ }^{*}(\%)$ \\
\hline PET & 247.9 & 251.9 & $55.4 \pm 2.8$ & & 200.3 & $48.4 \pm 0.3$ & 47.1 \\
NCP/MAWMA & 248.4 & 252.7 & $59.4 \pm 0.7$ & & 210.4 & $53.2 \pm 1.6$ & 50.5 \\
\hline
\end{tabular}

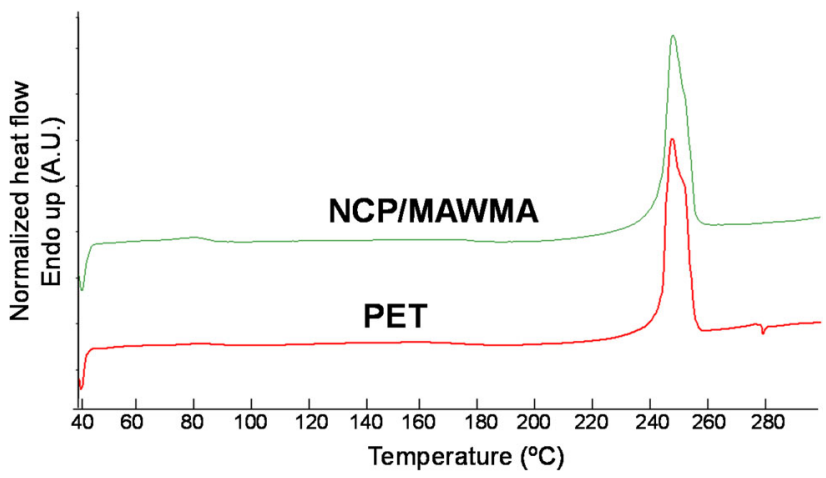

Figure 6. Thermograms of the heating process of PET and NCP/ MAWMA samples.

Table 10. Mechanical properties of PET and NCP/MAWMA multifilament obtained by dynamometric assays.

\begin{tabular}{lccc}
\hline Sample & Linear density (tex) & Tenacity $(\mathrm{cN} /$ tex $)$ & Elongation (\%) \\
\hline PET & $16.0( \pm 1.3 \%)$ & $35.9( \pm 4.4 \%)$ & $23.1( \pm 9.6 \%)$ \\
NCP/MAWMA & $16.4( \pm 5.5 \%)$ & $24.3( \pm 4.7 \%)$ & $23.0( \pm 7.3 \%)$ \\
\hline
\end{tabular}

post-thermal stabilization processes (same processes for both samples) of the yarns that imprinted two different stages of crystallinity into the multifilaments. Concomitantly, melting enthalpy $\left(\Delta \mathrm{H}_{\mathrm{m}}\right)$ of NCP/MAWMA multifilament increased $6.7 \%$ respect PET which was a significant change to take in account for further dyeing or other finishing processes of the yarns. Then, when samples were cooled down, crystallization points $\left(\mathrm{T}_{\mathrm{c}}\right)$ were spotted at $200.3^{\circ} \mathrm{C}$ and $210.4^{\circ} \mathrm{C}$ for PET and NCP/MAWMA, respectively (Figure 6). These differences resulted from the differences in composition of the samples, assuming that NCP systems needed less energy (temperature) to crystallize due to the effect of NPs and MAWMA in PET matrix rather than neat polymer. Crystallization enthalpies $\left(\Delta \mathrm{H}_{\mathrm{c}}\right)$ denoted a slightly increase in the NCP respect to PET, corroborating that the previously observed improvement of the crystallization properties of NCP/MAWMA, was now extensive in the multifilament form. Additionally, results of crystallinity $\left(\chi_{c}\right)$ confirmed the nucleating effect or crystallization capacity of SiC on PET.

Finally, dynamometric assays were developed to determine mechanical properties of the samples. Linear density spotted nearly the same for PET and NCP/MAWMA even after the inclusion of NPs and dispersing agent, which confirmed the good homogeneity of the NCP sample (Table 10). Regardless, tenacity decreased $\sim 32 \%$ in NCP/ 
MAWMA respect pure polymer, pointing to a decrease of the tensile strength. This phenomenon was also observed in a previous work and was related with the reduction of the intrinsic viscosity of the matrix of the polymer as consequence of inclusion of the NPs, the exposure to high temperature, shear rate and long residence time of the extrusion process (Riba-Moliner et al., 2020). Even though, elongation kept almost constant because the increase of the plasticity by the addition of the dispersing agent.

\section{Conclusions}

The evaluation of the effect of different dispersing agents in $\mathrm{PET} / \mathrm{SiC}$ systems has been explored towards the production of textile multifilament NCP. Firstly, non-isothermal crystallization process of NCP systems at different cooling rates has been analysed with the aim to investigate homogeneity, dispersion and crystal growth of samples with MAWMA, PSEMA and AW as dispersing agents.

DSC technique has revealed that $\mathrm{SiC}$ NPs would direct the non-isothermal crystallization of the nanocomposited systems of PET/PSEMA and PET/AW, meanwhile in MAWMA-based NCP, the dispersing agent may guide the process by itself. This observation, together with the appearance of no substantial changes in Tc values respect the pure polymer, has suggested a higher compatibility of the nanoparticles in the NCP/MAWMA system respect its homologues. The fact of the lipophilic character of MAWMA would also have contributed in the resulting higher compatibility of that NCP system. Furthermore, all samples have been corroborated to follow a crystallization process according to Avrami's model. In this sense, the inclusion of $\mathrm{SiC}$ has not remarkably modified $n$ values for all NCP samples and has suggested a crystallization process followed a growth of heterogeneous spherulites in $3 \mathrm{D}$. In the case of $Z t$ parameter, a significant increase in the presence of $\mathrm{SiC}$ compared to pure PET has been observed in all cases. However, over all samples, NCP/MAWMA showed the lowest values of $Z t$, even at the highest studied rates $\left(20^{\circ} \mathrm{C} /\right.$ $\min )$. In concordance with these observations, $t_{1 / 2}$ of $\mathrm{NCP} /$ MAWMA has denoted a different evolution respect the rest of NCPs, adopting a value of $0.53 \mathrm{~min}^{1 / 2}$ when was cooled at $20^{\circ} \mathrm{C} / \mathrm{min}$, essentially the same value as the found for PET.

Thus, a modified ester of montanic acid with multifunctional alcohols, MAWMA, has revealed as the dispersing agent that has induced to an improvement of the crystallization process respect the other proved nanocomposited systems. The fact that NCP/MAWMA follows a similar tendency in terms of non-isothermal crystallization behaviour as pure PET is very relevant in the industry of thermal processing of composites because suggests the possibility to process the NCP as pure polymer with similar results. Due to that, the evaluation of the effect of the concentrations of NPs and dispersing agent to the NCP has been analysed.

Regarding the effect of $\mathrm{SiC}$ and MAWMA concentrations on the NCP, the presence of the dispersing agent decreased Tg values, indicating that the plasticity of the system has increased. Consequently, samples with $2 \%$ of $\mathrm{SiC}$ and $2 \%$ of
MAWMA content presented a scenario with higher miscibility and balanced thermal behaviour respect the others. For this reason, hybrid multifilament NCP/MAWMA with a composition of $2 \% \mathrm{SiC}$ and 2\% MAWMA has been prepared and studied.

Furthermore, the determination of the molecular weight of the NCP/MAWMA filament has shown a decreasing of $\overline{\boldsymbol{M}_{\boldsymbol{n}}}$ and $\overline{\mathrm{M}_{\mathrm{w}}}$ respect pure PET filament which has been correlated with the sensitivity of the sample to the extrusion processing and the presence of NPs which have affected as well the viscosity of the sample. Additionally, two melting points in the thermal analysis of the multifilament have appeared. The similarity between those values has been attributed to the spinning and post-thermal stabilization processes that provide two different stages of crystallinity to the sample. $\Delta \mathrm{H}_{\mathrm{c}}$ has resulted in a light increase for NCP/ MAWMA respect to PET, corroborating the previous results and showing an improvement of the crystallization properties of the hybrid multifilament.

The determination of the mechanical properties by dynamometric assays has shown a similar linear density between PET and NCP/MAWMA which has allowed to confirm the good homogeneity of the nanocomposited sample. Even though, the tenacity of the NCP/MAWMA decreased 32\% compared to neat polymer, presumably indicating an associated decrease of the tensile strength. This fact may be related with the decrease of the previously observed molecular weight. Regardless, this decreasing is still within the parameters of using of textile PET and, extensively NCP/ MAWMA as filament.

In summary, this study has presented the production of a new hybrid multifilament based on $\mathrm{PET} / \mathrm{SiC}$ with an ester of montanic acid (MAWMA) as dispersing agent with thermal properties similar to pure PET. A well-dispersed and homogeneous NCP/MAWMA multifilament sample that can be thermally processed similarly to PET is very relevant from the point of view of the processing industry because it allows the possibility of working with similar conditions to pure polymer and produce nanocomposited materials without basically the loss of their properties.

\section{Ackowledgements}

Authors thank to the companies ANTEX, S.L. and La Seda de Barcelona for providing the different polyester pellets and, Clariant for providing the dispersing agents and to IQAP Masterbatch Group S.L. for the production of the hybrid multifilaments. Finally, authors acknowledge Mrs. Carmen Escamilla and Mrs. Montserrat García for their support in the experimental work.

\section{Disclosure statement}

No potential conflict of interest was reported by the author(s).

\section{Funding}

This work was supported by the Ministry of Science and Innovation of Spain under the Project MAT2010-20324-CO2-01; National Secretary of Higher Education Science Technology and Innovation of Ecuador (SENESCYT) under the Master's Fellowship No. 2015-AR2Q8981. 


\section{ORCID}

Marta Riba-Moliner (iD http://orcid.org/0000-0003-0001-2055

\section{References}

Agarwal, G., Patnaik, A., \& Sharma, R. K. (2013). Thermo-mechanical properties of silicon carbide filled chopped glass fiber reinforced epoxy composites. International Journal of Advanced Structural Engineering, 5(1), 21.

Avrami, M. (1939). Kinetics of phase change. I General theory. Journal of Chemical Physics., 7(12), 1103-1112.

Avrami, M. (1940). Kinetics of phase change. II transformation-time relations for random distribution of nuclei. Journal of Chemical Physics., 8(2), 212-224.

Avrami, M. (1941). Granulation, phase change, and microstructure kinetics of phase change. III. Journal of Chemical Physics., 9(2), 177-184.

Bhattacharjee, A., \& Nanda, B. K. (2017). Effect of silicon carbide as filler reinforcements on the mechanical and damping properties of Glass fiber/epoxy composites. Proceedings of COPEN 10 India

Cayuela, D., Cot, M., Algaba, I., \& Manich, A. M. (2016). Effect of different dispersing agents in the non-isothermal kinetics and thermomechanical behavior of $\mathrm{PET} / \mathrm{TiO}_{2}$ composites. Journal of Macromolecular Science, Part A, 53(4), 237-244.

Cheng, S. Z. D., \& Jin, S. (2002). Chapter 5 - crystallization and melting of metastable crystalline polymers. in Applications to polymers and plastics (vol. 3, pp. 167-195) (ed. S. Z. Cheng, D. B. T.-H. of T. A. and C.) Elsevier Science B.V.

Deng, Y., Li, J., \& Nian, H. (2018). Polyethylene glycol-enwrapped silicon carbide nanowires network/expanded vermiculite composite phase change materials: Form-stabilization, thermal energy storage behavior and thermal conductivity enhancement. Solar Energy Materials and Solar Cells, 174, 283-291.

Gacén Esbec, I. Modificación de la estructura fina de la fibras de PET en el termofijado y en su tintura posterior. Tintura competitiva de sustratos termofijados a temperaturas vecinas. (Universidad Politécnica de Cataluña, (2004).

Krikorian, V., \& Pochan, D. J. (2004). Unusual crystallization behavior of organoclay reinforced poly(l-lactic acid) nanocomposites. Macromolecules, 37(17), 6480-6491.

Manich, A. M., Cot, M., Algaba, I., \& Cayuela, D. (2015). Effect of the presence of an ester of montanic acids with multifunctional alcohols in the composites of titanium dioxide nanoparticles with poly (ethylene terephthalate) in their non-isothermal crystallization. Journal of Macromolecular Science Part A, 52(9), 770-777.

Mullaikodi, S. M., Shanmugasundaram, K., Rao, V. S., \& Rengarajan, S. (2019). Synthesis, characterization and machinability studies on thin hybrid composites with $\mathrm{SiC}$ nano particles. Materials Research Express , 6(6), 065321.

Patnaik, T. K., \& Nayak, S. S. (2018). Development of silicon carbide reinforced jute epoxy composites: physical, mechanical and thermomechanical characterizations. Silicon, 10(1), 137-145.

Pooley, M. A., Anderson, D. M., Beckham, H. W., \& Brennan, J. F. (2016). Engineered emissivity of textile fabrics by the inclusion of ceramic particles. Optics Express, 24(10), 10556-10564. https://doi. org/10.1364/OE.24.010556

Riba-Moliner, M., Mijas, G., Sánchez-Loredo, M. G., \& Cayuela, D. (2020). Obtaining of a PET-CaF2 hybrid multifilament: non-isothermal crystallization studies. Polymer Testing., 86, 106449.

Sánchez-Leija, R. J., Riba-Moliner, M., Cayuela-Marín, D., DomínguezEspinós, O., \& Sánchez-Loredo, M. G. (2014). Surface effect of two different calcium fluoride fillers on the non-isothermal crystallization behavior of poly(ethylene terephthalate). Journal of Macromolecular Science Part B, 53(2), 173-190.

Sánchez-Solís, A., Romero-Ibarra, I., Estrada, M. R., Calderas, F., \& Manero, O. (2004). Mechanical and rheological studies on polyethylene terephthalate-montmorillonite nanocomposites. Polymer Engineering \& Science, 44(6), 1094-1102.

Serenko, O. A., Roldughin, V. I., Askadskii, A. A., Serkova, E. S., Strashnov, P. V., \& Shifrina, Z. B. (2017). The effect of size and concentration of nanoparticles on the glass transition temperature of polymer nanocomposites. RSC Advances., 7(79), 50113-50120.

Shen, D., Zhan, Z., Liu, Z., Cao, Y., Zhou, L., Liu, Y., Dai, W., Nishimura, K., Li, C., Lin, C.-T., Jiang, N., \& Yu, J. (2017). Enhanced thermal conductivity of epoxy composites filled with silicon carbide nanowires. Scientific Reports, 7(1), 2606 https://doi.org/ 10.1038/s41598-017-02929-0

Shin, Y., \& Park, Y. (2018). Preparation and application of polymercomposited yarn and knit containing CNT/ceramic. Clothing \& Textile Research Journal, 36(1), 3-16.

Xue, W., Chen, P., Wang, F., \& Wang, L. (2019). Melt spinning of nano-hydroxyapatite and polycaprolactone composite fibers for bone scaffold application. Journal of Materials Science , 54(11), 8602-8612.

Yang, Z., Peng, H., Wang, W., \& Liu, T. (2010). Crystallization behavior of $\operatorname{poly}(\varepsilon$-caprolactone)/layered double hydroxide nanocomposites. Journal of Applied Polymer Science, 116, NA-2667. 